\title{
Publisher's Note: Critical wind speed at which trees break [Phys. Rev. E 93, 023001 (2016)]
}

\author{
E. Virot, A. Ponomarenko, É. Dehandschoewercker, D. Quéré, and C. Clanet
}

(Received 23 November 2016; published 30 November 2016)

DOI: 10.1103/PhysRevE.94.059907

This paper was published online on 2 February 2016 with a typographical error on page 1 . On page 1, right-hand column, the sixth line of the third paragraph should read as "frequency of the rod $f \simeq 0.56 \times \sqrt{E I / \rho_{s} S} / L^{2}(\mathrm{in} \mathrm{Hz})$, where $\rho_{s} \ldots$.". The paper has been corrected as of 17 November 2016. The text is incorrect in the printed version of the journal. 